\title{
AN ABSTRACT BOREL DENSITY THEOREM
}

\author{
MARTIN MOSKOWITZ ${ }^{1}$
}

\begin{abstract}
In this paper an abstract form of the Borel density theorem and related results is given centering around the notion of the author's of a (finite dimensional) "admissible" representation. A representation $\rho$ is strongly admissible if each $\Lambda^{\prime} \rho$ is admissible. Although this notion is somewhat technical it is satisfied for certain pairs $(G, \rho)$; e.g., if $G$ is minimally almost periodic and $\rho$ arbitrary, if $G$ is complex analytic and $\rho$ holomorphic. If $G$ is real analytic with radical $R, G / R$ has no compact factors and $R$ acts under $\rho$ with real eigenvalues, then $\rho$ is strongly admissible. If in addition $G$ is algebraic/ $\mathbf{R}$, then each $\mathbf{R}$-rational representation is admissible. The results are proven in three stages where $V$ is defined either over $\mathbf{R}$ or $\mathbf{C}$.

If $\rho$ is a strongly admissible representation of $G$ on $V$, then each $G$-invariant measure $\mu$ on $\mathcal{G}(V)$, the Grassmann space of $V$, has support contained in the $G$-fixed point set.

If $\rho$ is a strongly admissible representation of $G$ on $V$ and $G / H$ has finite volume, then each $H$-invariant subspace of $V$ is $G$-invariant.

If $G$ is an algebraic subgroup of $\mathrm{Gl}(V)$ and each rational representation is admissible, then $H$ is Zariski dense in $G$.
\end{abstract}

The Borel density theorem [1] states that if $G$ is a semisimple linear algebraic group/ $\mathbf{R}$ and $H$ is a discrete, or more generally a Euclidean closed subgroup such that $G / H$ has finite volume (or more generally has property $S$ ) then the algebraic (Zariski) hull $H^{\sharp}$ of $H$ equals $G$. In [4] I proved certain generalizations of the Borel density theorem in various forms. Principally this was done for minimally almost periodic groups (Furstenberg's case [3]), complex analytic linear groups (done independently by a different method by S. P. Wang [5]) and real analytic linear groups $G$ with radical $R$ and with the property that $G / R$ has no compact factors, $R$ acts with real eigenvalues and $H$ is a lattice in $G$. While there was a certain underlying unity to these results the methods seemed, on the surface, to be ad hoc. Relying heavily on [4] we present here an abstract form of the theorem which applies to all these cases simultaneously, gives the new result contained in Theorem $\mathrm{D}$, and which in addition proves a generalization of the last-named result of [4]. Finally, the results are now in a form where they could be directly applied to other situations.

Received by the editors November 8, 1978.

AMS (MOS) subject classifications (1970). Primary 20G20, 22E40, 22E15, 57E25.

Key words and phrases. Algebraic linear group, complex analytic group, radical, Levi factor, homogeneous space of finite volume, Zariski density, support of a finite $G$-invariant measure, $G$-fixed point set, Grassmann manifold.

${ }^{1}$ This research was partially supported by the National Science Foundation Grant \#MPS75-08268. 
In what follows, $G$ will be a locally compact group, $\rho$ a continuous finite dimensional real or complex linear representation of $G$ on $V, \mathcal{G}(V)$ denotes the Grassmann space of all subspaces of $V$ and $G \times \mathcal{G}(V) \rightarrow \mathcal{G}(V)$ will denote the induced action from $\rho$ on $\mathcal{G}(V)$. If $G \times X \rightarrow X$ is any action of $G$ on a space $X$ then $X_{\mathrm{fix}}$ or $X_{\mathrm{fix}, G}$ denotes the set of $G$-fixed points while $X_{c}$ or $X_{c, G}$ denotes the $G$-bounded points of $X$; that is those with compact $G$-orbit closures.

An examination of the results of [4] leads to the following definition. We shall say a representation $\rho$ of $G$ is admissible ${ }^{2}$ if there is a family of subgroups $\left\{H_{i}: i \in I\right\}$ which generate $G$ and such that each restriction $\rho_{i}=\rho \mid H_{i}$ has the following properties.

(i) $V_{c, \rho_{i}, H_{i}}=V_{\text {fix, } \rho_{i}, H_{i}}$.

(ii) $H_{i}$ has no closed subgroup of finite index.

(iii) For each $\left(\rho_{i}, H_{i}\right)$ invariant subspace $W$ of $V$ either $\rho_{i}$ acts on $W$ by scalars or else the function $g \mapsto \operatorname{det}(g \mid W) /\|g \mid W\|^{\operatorname{dim} W}$ vanishes at $\infty$ on $\rho\left(H_{i}\right)$, where \|\| is any convenient Banach algebra norm on End $W$.

We shall say that $\rho$ is strongly admissible if each $r$ th-exterior power $\Lambda^{r} \rho$ acting on $\Lambda^{r} V$ is admissible for $r=1, \ldots, \operatorname{dim} V$.

The importance of this notion is illustrated by the following theorem which is a slight modification of (1.11) of [4].

TheOREM A. If $\rho$ is a strongly admissible representation of $G$ on $V$ then each $G$ invariant measure $\mu$ on $\mathcal{G}(V)$ has supp $\mu \subseteq \mathcal{G}(V)_{\text {fix }}$.

We now give sufficient conditions for representations to be admissible.

THEOREM B. If

(1) $G$ is minimally almost periodic then any continuous representation $\rho$ is admissible,

(2) $G$ is complex analytic then any holomorphic $\rho$ is admissible. In particular all such representations are strongly admissible.

(3) Suppose $G$ is a real analytic group with $G / R$ having no compact factors and $\rho$ is a representation with the property that $R$ acts with real eigenvalues then $\rho$ is strongly admissible.

(4) Let $G$ be a real linear algebraic subgroupof $\mathrm{Gl}(V)$ which is Euclidean connected, such that $G / R$ has no compact factors and $R$ acts with real eigenvalues then each $\mathbf{R}$-rational representation $\rho$ is admissible.

The first three statements were proven in [4]. To prove (4) we note that since $\rho$ is an analytic representation: $G \rightarrow \mathrm{Gl}(W)$ we know that $\rho(R)=\operatorname{rad}$ of $\rho(G)$ and that $\rho(G) / \rho(R)$ has no compact factors. By (3) it is sufficient to see that $\rho(R)$ acts with only real eigenvalues on $W$. Since $R$ is a connected soluble algebraic group acting with real eigenvalues on $V, R$ is simply connected by (3.2)a of [4]. But $\rho$ is R-rational and by Lie's theorem we may

\footnotetext{
${ }^{2}$ We take the liberty of using the term admissible even though it is used in other contexts not completely disjoint from the present paper, see e.g. Harish-Chandra.
} 
consider rational characters $\chi$. Since $R$ is simply connected $\chi$ takes real values by (3.2)b of [4].

COROllary. The conclusion of Theorem A holds in Cases (1), (2) and (3).

Hereafter, we assume the locally compact group $G$ has a closed subgroup $H$ and $G / H$ has a finite $G$-invariant measure. Using Theorem $A$ we have as in [4]:

Theorem C. If $\rho: G \rightarrow \mathrm{Gl}(V)$ is strongly admissible then each $H$ invariant subspace of $V$ is automatically $G$-invariant.

In particular, by Theorem B it follows that the conclusion of Theorem $\mathbf{C}$ holds in Cases (1), (2) and (3) of Theorem B.

Via (2.4), (2.5) and (2.6) of [4] one has under the assumptions of Theorem C:

COROllary. If $\rho$ is irreducible so is $\rho \mid H$, the linear span of $\rho(G)$ equals that of $\rho(H)$ and the centralizer of $\rho(H)$ in End $V$ equals that of $\rho(G)$.

TheOReM D. Let $G$ be a $k$-algebraic subgroup of $\mathrm{Gl}(V)$ where $k=\mathbf{R}$ or $\mathbf{C}$ and suppose each $k$-rational representation, $\rho$ is admissible. Then $H$ is Zariski dense in $G$.

Proof. Consider the algebraic sugroup $H^{\sharp}$ of $G$. There exists a $k$-rational representation $\rho$ of $G$ on $W$ such that $H^{\sharp}=\{g \in G$ which leave stable a line $l$ in $W$ \} [2]. In particular, $l$ is $H^{\#}$ stable and therefore $H$ stable. Since $\Lambda^{r} \rho$ is a $k$-rational representation if $\rho$ is, the hypothesis implies that each $\rho$ is strongly admissible. By Theorem $\mathrm{C}, l$ is $G$-stable. This means $H^{\sharp}=G$.

We see by Theorem D and Theorem B that since $\mathbf{C}$-rational representations are holomorphic that:

COROLlARY. If $G$ is minimally almost periodic or a complex analytic linear group or a real analytic linear group as in Theorem $\mathrm{B}(3)$ then $H^{\sharp}=G$.

The last statement generalizes (3.4) of [4] from lattices to arbitrary cofinite volume subgroups.

In closing we note that using algebraic geometry, a different generalization of the Borel density theorem was presented by S. P. Wang in [6].

ADDED IN PROOF. It has come to the attention of the author that M. S. Raghunathan has also given a proof of the density theorem in the same simple case. Very recently another variant of the density theorem, using ergodic theory, in which a number of the present author's results (but now for $S$-subgroups) are reproven has been given by $S$. Dani.

\section{REFERENCES}

1. A. Borel, Density properties for certain subgroups of semisimple groups without compact components, Ann. of Math. 72 (1960), 179-188.

2. ___ Linear algebraic groups, Benjamin, New York, 1969. 
3. H. Furstenberg, A note on Borel's density theorem, Proc. Amer. Math. Soc. 55 (1976), 209-212.

4. M. Moskowitz, On the density theorems of Borel and Furstenberg, Ark. Mat. (1) 16 (1978), 11-27.

5. S. P. Wang, Homogeneous spaces with finite invariant volume, Amer. J. Math. 98 (1976), 311-324.

6. On density properties of S-subgroups of locally compact groups, Ann. of Math. 94 (1971), 325-329.

Departmient of Mathematics, Graduate School and University Center, Ctty UniverSITY OF NEW YORK, NeW YORK, NeW YORK 10036 